\title{
Sistemas funcionales, organizaciones y membrecía: paradojas sobre la inclusión organización/sistema parcial en Chile
}

\section{Functional systems, organizations and membership: paradoxes about organization/partial system inclusion in Chile}

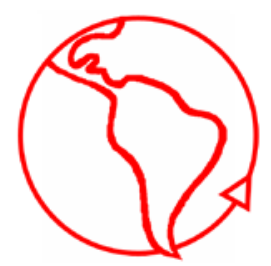

\section{Julio Labraña}

Departamento de Proyectos, Universidad Santa Federico Santa María, Chile

\section{Felipe Pérez- Solari}

Departamento de Historia, Universidad de Concepción, Chile

\section{Felipe Rivera}

Departamento de Educación, Fundación Integra, Chile

\section{Evelyn Campos}

Centro de Estudios de la Comunicación, Instituto de la Comunicación e Imagen, Universidad de Chile, Chile

\section{Resumen}

La diferencia entre inclusión y exclusión es uno de los rendimientos teóricos más reconocidos en la teoría sociológica propuesta por Niklas Luhmann. En este sentido, no se ha observado el problema de una correlación estricta entre la inclusión o exclusión organizacional y funcional, pues no se ha considerado con detenimiento la clausura operativa de cada uno de estos sistemas. El presente artículo aborda este problema mediante la hipótesis de que los criterios de inclusión en la membrecía de las organizaciones no adoptan necesariamente las formas probabilizadas por un sistema funcional en cuestión, sino que se definen autónomamente al esclarecer su impacto buscado. Posteriormente, se analiza el potencial explicativo de esta hipótesis frente a determinados fenómenos del contexto chileno, para concluir con indicaciones y preguntas derivadas de lo expuesto.

Palabras Clave: Inclusión; Exclusión; Diferenciación Funcional; Organizaciones; Membrecía

\begin{abstract}
The difference between inclusion and exclusion is one of the most renowned theoretical yields in sociological theory proposed by Niklas Luhmann. In this regard, it has not been observed the issue about a strictly correlated between inclusion and exclusion organizational and functional, because it has not been considered carefully the operative closure of each of these systems. The present article discuss this problem trough the hypothesis that the criteria for inclusion in the membership of the organizations do not adopt necessarily the probable forms the functional system in question, but must be defined autonomously to clarify its intended impact. Subsequently, it is analysed the explanatory potential of this hypothesis from certain phenomena in the Chilean context, to conclude with directions and questions arising from the above.
\end{abstract}

Keywords: Inclusion; Exclusion; Functional Differentiation; Organizations; Membership 


\section{Introducción}

La diferencia entre inclusión y exclusión ha sido considerada como uno de los pilares centrales de la teoría de sistemas sociales propuesta por Niklas Luhmann. El abandono de las tesis vinculadas al desarrollo y su remplazo por una forma de dos lados, que opera de manera ortogonal a sistemas funcionales autopoiéticos ha posibilitado la emergencia de nuevos enfoques en torno al problema de la evolución de la sociedad (Luhmann 1998a), la contraposición de la igualdad presupuesta frente a la desigualdad social (Nassehi 2011; Cadenas 2012) y la intervención basada tanto en políticas públicas (Mascareño 2005; 2011) como en la profesionalización del trabajo social (Robles 2002).

Asimismo, la teoría de las organizaciones ha recibido un nuevo impulso a partir de la integración de elementos de la teoría de sistemas (Luhmann 2007; Arnold 2008). La observación de las organizaciones como sistemas formados recursivamente por cadenas de decisiones ha permitido dar cuenta de los procesos que definen su horizonte de posibilidades, sin necesidad de importar elementos desde su entorno social, sino constituyéndolos a partir de la reproducción autopoiética de sus propias operaciones (Luhmann 2007).

Pese a la complejización que han sufrido los conceptos de inclusión y exclusión, organizaciones y sistemas funcionales, la estrecha relación entre ambos no ha sido suficientemente problematizada. Salvo por el desacoplamiento entre organizaciones y sistemas funcionales planteado por Morten Knudsen (2007), la literatura especializada, como veremos, no ahonda suficientemente en este tema. Es asumido de facto que las organizaciones forman parte de los sistemas funciones y los siguen en sus operaciones. El mismo Luhmann señala que las organizaciones se forman "dentro" de los sistemas funcionales y asumen el primado funcional del sistema parcial al cual pertenecen (Luhmann 2007: 667). En las escuelas se educa, en las clínicas se sana y en un banco se paga. Ciertamente pueden hacerse otras operaciones, pero todas éstas se subordinan a las directrices brindadas por un sistema funcional de referencia. Siguiendo el ejemplo anterior, en las escuelas se paga (medio dinero), pero esto se subordina a la realización de una programación educativa (mejor/peor); en las clínicas son utilizadas investigaciones científicas (verdadero/falso) pero siempre con una orientación al tratamiento del enfermo, y en los bancos pueden realizarse apelaciones morales (aprecio/desprecio), aunque no es relevante para las operaciones de la propia organización, pues la problematización de las decisiones se enmarca en el medio dinero.

En el contexto de diferenciación y autonomización de sistemas funcionales estas definiciones resultan insuficientes. La continuidad de la inclusión entre sistemas funcionales y sistemas organizacionales es un fenómeno contingente (no es necesario ni imposible), existiendo suficiente sustrato empírico para reflexionar teóricamente sobre el fenómeno. El que en escuelas y universidades se proceda por criterios educativos, y en hospitales según programaciones derivadas del sistema de la salud, es antes una posibilidad que un postulado derivado de la diferenciación.

A continuación sostendremos la hipótesis de que los criterios de organización en la membrecía no asimilan, en estricto rigor, las formas que un sistema funcional probabiliza en su operar, sino que por el contrario, se definen autónomamente de acuerdo a su impacto. La presentación de esta hipótesis comienza con aclaraciones conceptuales sobre qué se entiende por diferenciación funcional en la teoría de sistemas sociales y cómo se observa la diferencia entre inclusión y exclusión en los sistemas funcionales y organizacionales (1). Prosigue considerando las argumentaciones de Luhmann, para visibilizar en qué medida las organizaciones no operan 'dentro' sino 'a través' de los sistemas funcionales, orientando sus decisiones de acuerdo a directrices definidas en su propio encadenamiento de operaciones (2). Sobre esta base, la presentación de casos 
empíricos en Chile pretende dar cuenta de que este modelo de observación posee un mayor potencial explicativo (3). El artículo concluye con la evaluación de las consecuencias de la utilización de este enfoque en el análisis más preciso del carácter acumulativo de la exclusión.

\section{Diferenciación funcional: sistemas funcionales y organizaciones}

Para Luhmann la sociedad moderna puede definirse como funcionalmente diferenciada. En una utilización funcional-estructuralista de la teoría de sistemas y la cibernética de segundo orden (Brandão 2012), el sociólogo alemán define la diferenciación de un sistema como la reproducción, dentro del sistema, de la diferencia entre un sistema y un entorno. Luego, los sistemas crean en sus operaciones dos entornos: uno común a todos los subsistemas y uno separado para cada subsistema (Luhmann 1998a). El sistema político, por ejemplo, concibe la sociedad como entorno interno, tratando de organizar la relevancia política de motivos no políticos como lo 'público'.

En particular, la diferenciación de sistemas funcionales puede ser descrita como un incremento en su selectividad y negligencia. Los sistemas presuponen un mundo infinito a partir del cual crean un entorno dinámico, constituyéndose como altamente diferenciados y autónomos, operacionalmente cerrados, con su propia memoria y tipo de operación, sin posibilidades de tener un mecanismo central de coordinación (Luhmann 1994). La diferenciación funcional organiza sus comunicaciones en torno al cumplimiento de funciones especiales que serán abordadas al nivel de la sociedad: "cada sistema parcial reconstruye al sistema total -al que pertenece y co-realiza- a través de una diferencia propia (específica del sistema parcial) de sistema/entorno" (Luhmann 2007: 473). El funcionamiento autónomo de cada sistema funcional presupone las operaciones del resto de sistemas funcionales, lo que implica que la sociedad no puede conceder primacía absoluta a ninguna de ellas. Ni política, economía, educación u otro sistema funcional puede dirigir la evolución de la sociedad, en tanto toda irritación es mediada por las operaciones específicas del sistema funcional en cuestión (Mascareño 2011; Nassehi 2011).

Los sistemas funcionales se tratan como desiguales entre sí, pero tematizan a sus entornos asociados como iguales porque nada, excepto la función de cada sistema, justifica la discriminación. Dicho en los términos de Luhmann, la sociedad se orienta a la diferenciación funcional cuando:

Introduce la educación obligatoria para todos, cuando toda persona (noble o plebeyo, cristiano, judío o musulmán, niño o adulto) tiene el mismo status legal, cuando "el público" asume la función política de ser electorado, cuando a todo individuo se le reconoce la capacidad de elegir o no elegir un compromiso religioso y cuando todo el mundo, dados los recursos necesarios, puede comprar cualquier cosa y pretende conseguir cualquier ocupación. (Luhmann 1998a: 86)

En definitiva, cuando la posibilidad de inclusión se presupone como universal y la exclusión resulta de las operaciones de cada sistema.

En función de lo precedente, la diferencia entre inclusión y exclusión se define como la principal distinción producida por la diferenciación funcional. De acuerdo a esto, Luhmann establece un distanciamiento del abordaje otorgado al problema de la integración, que había sido conceptualizado tanto por la sociología de Parsons como por otros referentes. Hasta aquí el gran inconveniente observado -debido a sus presupuestos en torno a comportamientos normativos- tiene que ver con fomentar que la exclusión sea considerada como una desviación repelida por la integración. Inspirándose laxamente en el concepto de forma de Spencer-Brown (1972), Luhmann entiende la inclusión como una 
indicación de un lado marcado cuyo lado no marcado es la exclusión (Luhmann 1998b: 170-171). En consecuencia, toda operación actualiza un lado de inclusión opuesto a su concepto sombra, la exclusión, sin que en ningún caso puedan realizarse totalmente en la sociedad.

En una sociedad funcionalmente diferenciada, según Luhmann, la inclusión y la exclusión abandonan una regulación universal: la relevancia política de las personas se regula separadamente por el sistema político y si se utilizan influencias económicas, ello obedece a un cálculo en torno a la eficacia política de su reputación. Idéntico proceso es observable tanto en la formación de familias, realizada por los sistemas de la intimidad, como en la búsqueda de educación, monopolizado por organizaciones escolares y universitarias propias del sistema de la educación. Más aún:

La participación en el sistema económico se rige por medio de la propiedad y la renta; la participación en el sistema educativo, a través de la escuela, las calificaciones escolares y las titulaciones - y no (...) a través del Espíritu Santo (que en este caso quiere decir talento). (Luhmann 1998a: 177)

La definición realizada desde la teoría sobre la forma inclusión/exclusión es la de un metacódigo que funciona en forma ortogonal a los sistemas funcionales, señalando posibilidades limitadas de comportamientos de los individuos. No se apunta a su tematización ontológica o a su abordaje como entes trascendentales, sino que a su observación como artefactos comunicativos que operan mediante biografías individuales actualizadas en base a los requerimientos que los sistemas funcionales exigen para que sean incluidos en las prestaciones auspiciosas de sus funciones (Robles 2002). Para Luhmann, estar incluido en los sistemas funcionales significa tomar como relevantes a los seres humanos en la comunicación, es decir como "personas" (Luhmann 1998c: 238- 239), mientras que estar excluido implica una observación centrada en el cuerpo, donde la percepción y la ligereza priman (Luhmann 1998b; Nassehi 2011). No está excluido de la comunicación científica el individuo que produzca resultados científicos falsos, sino el individuo del que no se espera operación científica alguna, sea ésta verdadera o falsa. Lo mismo sucede en el caso de la educación, donde no está excluido el alumno que obtenga una mala calificación, sino el niño sobre el que no se apliquen las programaciones mejor/ peor, propias del sistema de la educación (Luhmann \& Schorr 1990).

Las operaciones de inclusión son reguladas en los sistemas funcionales por códigos y programas, en los sistemas organizacionales esto se consigue mediante cadenas de decisiones. En el caso de los sistemas funcionales, el binarismo del código permite que asuman una orientación al universalismo y la especificidad. El universalismo se refiere al ámbito de aplicación universal, que no se encuentra limitado por circunstancias externas, mientras que la especificidad apunta a la orientación hacia una única función (Luhmann 2007). En el caso del sistema de la economía, por ejemplo, éste observa toda irritación como comunicaciones basadas en la diferencia entre pago/ no pago, y no puede escoger guiarse por una distinción que no sea precisamente aquella. En el mismo sentido, si bien los experimentos de laboratorio están imbricados por variables ajenas al sistema científico (Kuhn 2010), en última instancia es el código (verdadero/falso) el que permite, mediante publicaciones, prescindir de la historia que ha conducido a cierto resultado a ser verdadero (Nassehi 2011).

El código opera en forma ortogonal a la diferencia inclusión y exclusión de los sistemas funcionales, de forma tal que estar incluido en las comunicaciones del sistema no refiere a una preferencia en el valor positivo sobre el valor negativo. Consecuentemente, todo lo que puede ser comprendido por el código aparece como contingente, como posible (Luhmann 2007). Un pago sólo es posible en tanto existe la posibilidad de no pagar, así como una ciencia que se regula por la verdad será incapaz de reflexionar sobre las 
condiciones que hacen esto posible. Luhmann señala que "el valor positivo simboliza siempre la capacidad de enlace para las operaciones específicas del médium, mientras que el valor negativo simboliza sólo la contingencia de las condiciones de la capacidad de enlace" (Luhmann 2007: 283).

A partir de lo anterior, la adscripción tanto en el valor positivo como en el valor negativo del código está mediada por la inclusión en los sistemas funcionales. Exclusión es, en este sentido, todo lo que no aparece en la pantalla de los códigos de los sistemas funcionales de la sociedad (Nassehi 2011). Inclusión, en cambio, es la regulación de las comunicaciones por un sistema funcional determinado. No es casualidad que el teórico alemán no asociara la exclusión con el valor negativo del código, sino con la ausencia de presupuestos simbólicos en la comunicación (Luhmann 1998b). La codificación excluye toda meta regla sobre la comunicación, pues ésta únicamente podría tomar posición en el valor positivo o negativo del sistema (Luhmann 2007).

El código de los sistemas funcionales excluye terceros valores (es blanco o negro sin que se acepten grises). Por tanto, toda posibilidad se convierte en una elección entre o-esto-olo-otro, con lo que se gana una pregunta de decisión clara, referente al código, que resulta idéntica para alter y para ego (Luhmann 2007). Al simplificar este paso del valor (+) al valor (-), a partir de una negación, el código mismo se convierte en una estructura invariable. A la vez se hace difícil, y ésta es una característica de la diferenciación funcional, que valores positivos (o negativos) de diferentes códigos se aglutinen entre sí (Luhmann 1994; 1998b; 2007). Ser rico no implica ser sabio ni poseer capacidad política para ser presidente, y ser pobre tampoco supone un status moral más elevado (Mascareño 2005).

En la medida en que estos códigos facilitan el paso hacia el otro valor, surge una libertad en el contexto de la operación que nuevamente debe restringirse a una semántica de criterios adicionales que determine en qué condiciones el valor positivo o negativo es adscrito correctamente. Estos condicionamientos son los programas (Luhmann 2007). Al contrario de los códigos, imposibles de variar, en el nivel de los programas olvidar y recordar es posible dependiendo de su utilización. Ni la teoría o métodos de la ciencia, ni los programas de inversión o consumo en la economía, ni las semánticas de historia común en el amor permanecen inconstantes en el tiempo, pero continúan subordinadas a los códigos de los sistemas funcionales respectivos de la ciencia (verdadero/falso), economía (pago/no pago) e intimidad (amor/no amor).

A diferencia de los sistemas de interacción y los sistemas funcionales, las organizaciones se definen por los criterios que seleccionan quienes pueden pertenecer a ellas. Antiguamente, las organizaciones tenían como finalidad brindar capacidades de supervivencia a los individuos, mediante la relación organización-trabajo. No obstante, esta idea debe ser matizada. En la Edad Media, las organizaciones eran agrupaciones que intentaban representar intereses de grupos que no necesariamente requerían de ellas para continuar sus propias operaciones. Ante la escasa diferenciación, las organizaciones pretendían representar intereses en el ámbito de la política y los privilegios (Luhmann 2007: 663).

En la modernidad, en cambio, las organizaciones son motores de solución de problemas. Ante el continuo aumento de la complejidad de la sociedad contemporánea, las organizaciones tienen la posibilidad de enfrentarse a las dificultades que ellas conllevan, asignándose la función de articular organización para enfrentar dicha complejidad. Bajo esta lógica, no es menor que a la sociedad contemporánea se le denomine “organizacional" (Rodríguez 2004). 
En palabras de Eisenstadt (1968: 21), existen tres criterios para caracterizar las organizaciones (él habla de asociaciones) en la sociedad moderna: 1) gran cantidad de ellas poseen funciones específicas; 2 ) existe división del trabajo entre las mismas y otras de finalidad solidaria y cultural, y 3) debilitamiento de la importancia del parentesco y las bases territoriales estrechas de las mismas asociaciones especializadas. Lo anterior no es conflictivo con la definición sistémica, pues las organizaciones se tematizan en relación a una función, aún cuando puedan operar con otras funciones, siempre hay una de ellas que prima. De este modo, se observa una división en el trabajo, pero en el sentido de la articulación medio- fines que la misma organización busca (Rodríguez \& Arnold 1991). Por último, ya no prima el parentesco y la territorialidad, ya que no es suficiente la familia para organizar la creciente complejidad, ni tampoco basta la relación organización/territorio para combatir los procesos entrópicos que implica su aumento (Luhmann 2007).

La operación fundamental de las organizaciones son las decisiones (Luhmann 2007), puesto que al comunicarlas permite que se siga decidiendo, conectándose esta relación con decisiones más específicas que se convierten en acción. Si se cumplen las decisiones, ello no significa dejar de decidir, sino simplemente continuar enfrentándose a la contingencia del sistema (Rodríguez 2012).

A partir de esta característica emergente de las organizaciones, se observa que éstas tienen una particular forma de tratar con la doble contingencia, que es distinta a la utilizada en los sistemas funcionales e interaccionales. Según afirma el propio Luhmann "cada cual puede actuar de otra manera y corresponder o no a los deseos de expectativas - aunque no como miembro de una organización". (Luhmann 2007: 657). Esto pues existe la posibilidad de perder la membrecía organizacional en caso de que un miembro se oponga a los criterios demarcados por la membrecía misma. Lo dicho anteriormente impacta en el tratamiento de la doble contingencia, pues la membrecía puede condicionarse no tan sólo al ingreso, sino también a la mantención del status de miembro de la organización (Luhmann 2007).

No obstante, las relaciones cara a cara no serán la base de articulación de la organización, por el contrario, las reglas explícitas de pertenencia, fijadas en normas, dirimirán el problema de la inclusión. Esto no quiere decir que en las organizaciones no existan sistemas de interacción, sabemos que se encuentran presentes, pero la espontaneidad y plasticidad propia de los sistemas de interacción - es decir, su posibilidad de interrumpir la distinción código/programa a placer, así como la de pertenencia/no-pertenencia- se subyuga a la existencia de reglas. En esta dirección, Robles (2006a: 10) - haciendo eco de lo propuesto por Schütz- sostiene que las dos condiciones para los sistemas de interacción son: 1) compartir un espacio que contenga a los cuerpos que participan; 2) intervenir en el tiempo social de la propia interacción como en la duración de ella. Nada más contrario a las organizaciones, que se nutren de roles a través de reglas y reductores de variedad, para que las decisiones efectivamente alcancen la finalidad por la cual fueron creadas. Por ello, la organización se articula en estructuraciones programáticas (tareas, puestos, redes, posiciones jerárquicas) definidas en su propia comunicación de decisiones, orientada a metas, y no en base a personas (Rodríguez \& Arnold 1991).

Como se ha señalado, las organizaciones son los únicos sistemas sociales que regulan sus mecanismos de inclusión/ exclusión por las decisiones que ellas mismas realizan. El estar incluido en una organización es ser partícipe, más allá del rol que se cumpla, de las comunicaciones de la organización. Si se pasa al otro lado de la distinción, es decir al lado de la exclusión, simplemente no se es observado (Robles 2010). Cabe aclarar que existe una distinción entre la exclusión que mencionamos (ser expulsado de la organización) a ser tematizado como excluido, pero incluido dentro de la pantalla (empresa de cobranzas). 
A diferencia de los sistemas funcionales, las organizaciones operan en la exclusión, no en la inclusión total, lo anterior simplemente porque su propia especificación no puede tematizar la inclusión total de los individuos (necesidad de criterios de membrecía), ni en ellas ni en todos los sistemas funcionales (Arnold 2008; Rodríguez 2004; Luhmann 2007; Robles 2006b; 2010). Simplemente, es imposible que una organización incluya a todas las personas dentro de sus propias operaciones y cuando aspira a hacerlo debe poseer organizaciones de organizaciones que sean capaces de llevarlo a cabo, aunque siempre de manera artificial (v. gr. el Estado, aunque para eso deba existir la condición de vivir o tener relación con él) (Torres Nafarrate 2004).

Para diferenciarse de sus miembros, la pertenencia en las organizaciones se define en términos de requisitos, competencias y aportes. Para concretar sus fines, la organización aplica una drástica reducción de posibilidades (Arnold 2008), no estando esto lejos de los llamados "reductores de variedad" de las primeras formulaciones de la teoría general de sistemas (Johansen 2007). Los acoplamientos de las organizaciones con la membrecía tienen directa relación con el desempeño en el puesto de trabajo, lo que se puede premiar/castigar, al tiempo que observar cómo deben ser los comportamientos de ánimo en el mismo desempeño de la función laboral asignada (Arnold 2008).

Las formas de inclusión en una organización no son siempre las mismas. La jerarquía se distribuye de casilla-en-casilla, siendo las decisiones capaces de alcanzar a todo el sistema por medio de esta división (Luhmann 2007). En esta dirección, no todos tienen la misma injerencia en la cadena de decisiones. En la idea de "público" de la política (Torres Nafarrate 2004), los miembros adscritos al Estado no tienen entrometimiento en las formas en que la administración los tematiza, dándose una relación de prestación de servicios, pero no de posibilidad de intervenir en cómo se dan esos servicios.

Las organizaciones dan operatividad tanto a los códigos como a los programas de los sistemas funcionales. Por lo cual, deben especificar funciones societales de forma clara para ser asumidas por ellas. Aunque la sociedad señala las premisas que deben ser autoseleccionadas y ordena sus entornos relevantes, respeta la autoselección organizacional (Rodríguez 2004). Asimismo, las organizaciones son los únicos sistemas sociales capaces de comunicarse con sus entornos, esto porque presuponen la autopoiesis sobre sus decisiones, no contradiciendo con ello su propia clausura operativa (Luhmann 2007).

Según Luhmann (2007: 667), las más grandes e importantes organizaciones (económicas, estatales, políticas, escolares, científicas jurídicas y legislativas) se forman 'dentro' de los sistemas funcionales, asumiendo el primado funcional por ellos asignado, mas debe quedar claro que ningún sistema funcional puede alcanzar su unidad sólo como organización. Los sistemas organizacionales asumen el primado de la función, y su código binario, del sistema funcional que 'representan', aunque haciendo concesiones a otras funciones. Con estos requisitos, las organizaciones relacionan sus operaciones con el sistema funcional representado, volviéndose identificables como escuelas, bancos o tribunales. Su mundo propio se activa mediante la distinción programa/ decisiones.

Los sistemas funcionales consideran la inclusión como algo normal., En otras palabras, los sistemas funcionales no excluyen a nadie a priori como existía en las diferenciaciones anteriores de la sociedad (Luhmann 1998a). Contrariamente, las organizaciones excluyen a todos, excepto a sus miembros, de una manera tremendamente selectiva, siendo esto último decisivo para la función, ya que solamente con organizaciones formadas internamente, pueden los sistemas funcionales regular su apertura a todos y tratar a las personas de forma distinta, aún cuando no todos tienen la capacidad de acceso. Más que haber una relación completamente jerárquica entre sistema funcional y organizacional 
aparece el concepto de red (Luhmann 2007). Ergo, "las organizaciones desarrollan una dinámica propia que es asumida en el sistema funcional mediante procedimientos de observación de segundo orden" (Luhmann 2007: 671).

2. Relaciones entre sistemas funcionales y organizaciones. Inclusión/exclusión y participación en el código funcional mediante sistemas organizacionales. Inclusión y Exclusión: desacoplamiento entre organizaciones y sistemas funcionales

Las organizaciones orientan su estructura de acuerdo a un ruido originario (Arnold 2008), que remite al fin operativo al que intenta responder la cadena de decisiones. Es desde aquí donde se promueven las comunicaciones con respecto a un fin. Por tanto, una problemática definitoria es la que guía las decisiones organizacionales y las preguntas sobre la problemática en cuestión. Según Arnold "sólo constituyendo su problema la organización objetiva sus procesos, lo cual le permite, simultáneamente, autodiseñarse" (Arnold 2008: 98). Esto es coherente con la operatividad de la distinción sistema/ entorno, puesto que la organización de estructuras - en este caso, cadenas de decisiones- está articulada exclusivamente por las operaciones del sistema.

Cuando una red recursiva de decisiones identifica las observaciones basales, que van a guiar este proceso, puede jerarquizar la consecución de objetivos desde criterios de criterios. Summa summarum, por medio de sus programas racionalizadores el sistema está en condiciones de reducir complejidad, dividiendo y programando decisiones que resultarán en una operación soberana de expansión/contracción de sus entornos. Los criterios de membrecía se determinan desde las comunicaciones del sistema, que de forma ordenada se concatenan por medio de las decisiones. Es aquí cuando la viabilidad deja de sostenerse por su criterio de demarcación originario, transformándose en impacto al contar con la capacidad de ponderar el éxito/fracaso de los motivos que dieron origen a la organización, enfocándose lo demarcado en la sujeción a las reglas reflexivamente trazadas (Arnold 2008).

En un interesante artículo, Knudsen (2007) propone que las teorizaciones sobre la relación entre organizaciones y sistemas funcionales no son suficientes para analizar la complejidad de los acoplamientos entre ambas. La propuesta refiere a que las organizaciones están acopladas a los sistemas funcionales en concordancia a cómo éstas desparadojizan sus decisiones.

El autor difiere de la idea de Luhmann sobre que las organizaciones más importantes se forman con los sistemas funciónales, así como que están asignados a una sola función desde sus operaciones hacia los sistemas funcionales y es así como se asigna a la sociedad por modo de la diferenciación funcional (Luhmann 2007: 840-847, 2000: 405 citado de Kudsen 2007: 112). Pero, ¿qué hacer ante el problema teórico de sostener que una organización opera en dos o más sistemas funcionales (más allá del concepto de 'red')? Siendo coherentes con la distinción sistema/ entorno, Knudsen se pregunta por los impactos que genera la diferenciación funcional. Si esta, dentro de sus despliegues, radicaliza la distinción sistema/entorno, deja solamente la posibilidad de relación no determinante entre los sistemas desde la posibilidad de los acoplamientos estructurales (Ziemann 2007). Un acoplamiento estructural permite respetar los procesos autopoiéticos de un sistema y, al mismo tiempo, variadas relaciones entre ellos. Desde el entorno un sistema puede generar ruido a otro sistema, irritando sus comunicaciones. Poniendo a Luhmann contra Luhmann: si las organizaciones operan dentro de los sistemas funcionales, la explicación sobre el cómo se acoplan a otros, por la necesidad de la cadenas de operaciones, queda opaca (Knudsen 2007).

La innovación teórica es la posibilidad de desparadojización de la cadena de decisiones de las organizaciones mediante expectativas de acoplamiento estructural con otros sistemas 
funcionales. Es decir, este tipo de sistemas sociales son capaces de tematizar la eventualidad de sus problemas de decisiones y su posible resolución, de acuerdo a criterios internos (Knudsen 2007). Retomando la idea de Arnold (2008) sobre la cadena de decisiones que transita de ruido originario a impacto, así como la potestad soberana de expandir/contraer sus entornos, no es de extrañar que los sistemas organizacionales reduzcan la contingencia de sus decisiones mediante acoplamientos estructurales con los sistemas funcionales.

La membrecía en las organizaciones se encuentra determinada por el mismo sistema. Él decide quién está dentro/fuera, así como cuáles son los criterios de pertenencia que deben cumplirse para que esto se lleve a cabo. Las reglas y los miembros pueden alterarse, pero con el fin de mantener la posibilidad de relacionar los miembros con las reglas y viceversa (Luhmann 2005). Volviendo a la idea original de Arnold, los criterios de membrecía se definen en la medida en que el propio sistema sentencie quién está en el sistema y quién no.

Recapitulando, la cadena de decisiones de las organizaciones y la determinación de quién es miembro de las mismas puede señalar que estar incluido es distinto para los sistemas organizacionales y funcionales. En otras palabras, la inclusión en una organización no implica participar como miembro, necesariamente, en la desparadojización de la comunicación de decisiones por medio de los acopamientos estructurales organización/sistema funcional. Menos aún, si la inclusión es en el "rol de público", más que en el "rol de desempeño" (Cadenas 2012). Más categóricamente, no todos los miembros participan de la absorción de incertidumbre de la cadena de decisiones, puesto que la jerarquización impide que esto ocurra (Torres Nafarrate 2004).

En consecuencia, la inclusión en el rol de público en una organización que se acopla estructuralmente a sistemas funcionales, puede ser completamente distinta a otra con el mismo ruido originario.

\section{Hiperautonomía organizacional y sistemas funcionales: Educación y salud en Chile}

La hipótesis precedente señala que la desparadojización de las decisiones en las organizaciones no presenta una correspondencia unívoca, sea en la inclusión o exclusión, con el sistema funcional al cual tradicionalmente son asociadas. En el panorama chileno encontramos fenómenos relativos al encadenamiento de decisiones, a través de fórmulas de impacto económicas, tanto en la educación como en la salud, referencias empíricas que permiten destacar el modo de procesamiento de las decisiones.

En el caso de la educación, lo anteriormente expuesto se puede identificar en los criterios que guían a algunas organizaciones educativas en los niveles de enseñanza básica y media, en relación al tratamiento de las calificaciones y su posterior inflación. Como señaló Luis Riveros (2004), ex Rector de la Universidad de Chile (1998-2006), las distorsiones en la distribución de notas no presentan correlación con el rendimiento efectivo de los estudiantes en las universidades. En el mismo sentido, es posible señalar que los mejores alumnos de liceos públicos obtienen un puntaje significativamente menor en las pruebas de ingreso a la educación superior que sus pares de colegios privados (Fernández 2011). Asimismo, existe una presión sobre las escuelas de alumnos de menores ingresos por calificar con notas altas a exámenes mediocres. Se busca que la subvención otorgada por el Estado no se pierda, a la vez que sean evitados conflictos con las familias de los alumnos o la institución pierda competitividad frente a las otras. Si bien la primacía de la programación educativa mejor/ peor permanece como criterio rector en la toma de decisiones de la organización, su aplicación se vuelve más laxa. La decisión de aplicarla o no aplicarla se toma en función de los impactos económicos buscados, sin que estos sean, necesariamente, los mismos de la comunicación educativa. 
Parte importante de los criterios en las organizaciones de la educación superior se definen también de esta manera. En Chile son varias las universidades, institutos profesionales y centros de formación técnica en los que se realiza una autodescripción que diferencia las vías monetarias de ingreso como miembro de la organización y la relevancia de la programación educativa una vez incluido. Regula la inclusión la capacidad de costear del estudiante (pago/no pago), y sólo una vez dentro de la organización se aplican programaciones tradicionales de la educación (mejor/peor), aunque subordinadas todavía a la persecución de intereses económicos. De forma paradójica, un joven puede no ingresar a la educación superior, pese a haber sido evaluado exitosamente en sus certificaciones académicas mientras que, en un sentido opuesto, un alumno puede no acreditarlas (esto es, ni siquiera operar en la distinción mejor/peor), pero mediante la utilización del medio dinero acceder a instituciones que lo evalúen académicamente.

Ahora bien, la estructuración jerárquica de este tipo de universidades, en relación al rol que cumplen los profesores, es mediada por contratos a honorarios que permiten criterios de flexibilización laboral. Fenómeno que es coherente con la superposición del código pago/no pago, en vez de una articulación contractual más estable que permitiría un mejor desempeño de las pautas funcionales que dicta el sistema educativo (CENDA 2011). Así, el alumno es tematizado como persona en la organización sólo mientras sea capaz de generar dinero y el profesor, como un funcionario que cumple un rol que permite la articulación programática de la distinción mejor/peor.

Es preciso trazar una separación en el nivel organizacional. Todas las universidades en Chile, pertenezcan o no al Consejo de Rectores, requieren de la cancelación de una matrícula y el pago de mensualidades. Esto puede hacer pensar que todas operan en el medio del dinero por sobre la educación. La diferencia entre ellas está, sin embargo, en que mientras las universidades del consejo utilizan obligatoriamente un mecanismo de ingreso académico expresado en una prueba de selección académica (mejor/peor), en el resto la actualización de esta posibilidad queda definida por el ruido originario transformado en el impacto buscado por la propia organización.

La paradoja mencionada no se reduce únicamente al sistema educativo, también es posible apreciarla en las codificaciones del sistema de la salud chileno y su distinción entre enfermo/no enfermo. Las instituciones de salud previsional (ISAPREs) operan como mecanismo privado de acceso a la salud, en oposición al fondo nacional de salud (FONASA) de carácter estatal. La forma de desparadojización de las decisiones es similar a la señalada anteriormente, esto es, no se corresponde con el sistema funcional al que hipotéticamente la organización pertenece. Como explica Rafael Caviedes, director ejecutivo de la Asociación de ISAPREs, los incrementos en los costos de los planes de salud se explican porque estas organizaciones funcionan en base al interés de los inversionistas en generar servicios que les permitan generar más dinero ${ }^{1}$. En este sentido, las programaciones propias del sistema de la salud derivadas del código enfermo/no enfermo son tematizadas en las organizaciones mediante fórmulas de impacto económicas.

La desparadojización de las decisiones en las organizaciones a partir de otros sistemas funcionales abre varias posibilidades que, ciertamente, son actualizadas en las ISAPREs. La

\footnotetext{
${ }^{1}$ Rafael Caviedes señala en entrevista para la radioemisora chilena "Radio Concierto": "El lucro, a mi juicio, no debería ser un tema en discusión por cuanto el lucro lo que genera justamente es interés de los inversionistas en generar servicios, generar empresas, que al final son los que están innovando en materia de salud, están generando empleos y son los que están dando mejores respuestas y el mejor servicio de salud lo está dando, justamente, el sector empresarial en nuestro país y eso es una realidad. Probablemente para la gente que esté en el sistema estatal de salud lo vea desde una perspectiva distinta pero... es la empresa privada la que está desarrollando el sistema de salud en nuestro país." (12 de junio de 2012)
} 
variación de precios en los planes de salud mediante la introducción de nuevas patologías, la negación a atender ciertas enfermedades costosas o el rechazo-acortamiento de licencias médicas parecen apuntar en esta dirección (Barría 2012). Por consiguiente, la coherencia en la cadena de decisiones médicas se supedita a la continuación de la comunicación económica en la organización ${ }^{2}$. El sistema del derecho probabiliza estas selecciones. Por ejemplo, para la anualidad entre julio de 2012 a junio de 2013, la Superintendencia de Salud de Chile calculó un reajuste del 2,15\% en los planes de salud, mientras que el aplicado por las ISAPREs, basado en sus propios cálculos de gastos, alcanzó el 2,4\%, siendo reconocido que, legalmente, estas pueden proceder en sus decisiones en forma autónoma en la normalización de precios (Ley N 19.496) ${ }^{3}$.

Las ISAPREs también desacoplan sus operaciones del sistema funcional al cual teóricamente pertenecen (salud), para adoptar criterios de orientación en sus decisiones que son propios de otras formas de comunicación (economía). Los criterios que utiliza para definir como relevantes a los seres humanos no se condicen con los del sistema funcional de la salud. En términos prácticos, esto queda en evidencia en los resultados de los reajustes en los planes de salud de las ISAPREs. Los afectados pueden aceptar pasivamente el aumento de costes, cambiarse a un plan con menos prestaciones o retirarse al sistema estatal de salud. En cualquiera de estos casos, la organización prosigue la desparadojización de su cadena de decisiones en torno al impacto económico de sus decisiones anteriores. La comunicación es tematizada en base a la diferencia enfermo/no enfermo, en tanto sea capaz de generar dinero en la organización. En el caso opuesto, es decir, que no sea capaz de encadenar decisiones orientadas por el medio del dinero, la comunicación pasa a situarse en la exclusión del sistema funcional de la salud.

\section{Conclusión}

Considerando los puntos expuestos, pareciera ser que la diferenciación funcional opera de forma hiperautónoma sin ser capaz de tematizar lo que los sistemas funcionales/organizacionales desean uno del otro. Si la política necesita irritar a la economía, debe hacerlo mediante los rangos que le permite el sistema del derecho e intentando mantener su función de tomar decisiones que vinculen colectivamente (Luhmann 2007; Torres Nafarrate 2004). La introducción de las organizaciones, en tanto le dan contenido operativo a los sistemas funcionales de la sociedad, no parece operar necesariamente por continuidad respecto de ellos. Más bien, la desparadojización en la comunicación de sus decisiones es lo que guía los acoplamientos estructurales entre estos y los sistemas funcionales.

La hipótesis que se ha presentado permite una observación más precisa de los mecanismos de inclusión y exclusión, pues separa estas distinciones en los niveles organizacional y funcional. De esta manera, se abandona el modelo de una matroska, donde las organizaciones estarían dentro de los sistemas funcionales, por uno que se asemeja más a las "mónadas" de Leibniz (1980), donde las organizaciones operan de forma discreta frente a los sistemas funcionales. En este sentido, parece plausible asociar esta formulación con observaciones que rescaten, desde la propia teoría de sistemas, el carácter acumulativo de la exclusión frente a la contingencia que define a la inclusión en los sistemas funcionales (Luhmann 1998b; Robles 2002, 2010; Mascareño 2005). Los beneficios de esta unión son evidentes. Dado que inclusión y exclusión son observaciones

\footnotetext{
${ }^{2}$ En esta dirección, tiene sentido la defensa del abogado de ISAPRE Colmena, Claudio Arellano Parker, a propósito de los costes diferenciados por edad y sexo en los planes de salud: "Si se analiza este factor a lo largo de sus vidas, no hay verdaderamente, entonces, una discriminación entre hombres y mujeres, niños o ancianos, sino sólo una anticipación y cuantificación estadística de las unidades de riesgo que generarán, de forma que cada uno de los afiliados a ISAPRE pague en base a esas unidades de riesgo, y sólo en base a ellas, sin más diferencias" (Arellano 2012).

${ }^{3}$ Ley que establece normas sobre protección de los derechos del consumidor. Nos referimos al artículo segundo, inciso f, que deja fuera de la observación de la ley las prestaciones de salud y materias relativas a su calidad y financiamiento.
} 
a definir por organizaciones y sistemas funcionales, no basta con señalar su presencia en uno de estos niveles para automáticamente asumir como presente un correlato en el siguiente. No basta con dar cuenta de las "pantallas" de los sistemas funcionales (Nassehi 2011), sino también observar las definiciones que las organizaciones realizan en su cadena de decisiones. A modo de proyección, cabría plantear la pregunta de qué es lo que ocurre cuando se está incluido en la membrecía de la organización y excluido del sistema funcional. Se presenta este planteamiento como temática abierta para posibles futuras investigaciones atingentes a los asuntos tratados en el presente artículo.

En la misma dirección, esta separación entre organizaciones y sistemas funcionales plantea importantes interrogantes acerca de qué es lo que define la relación que existe entre organización y sistemas funcionales. De igual manera, se abren nuevos flancos para problematizar algunas características de la sociedad contemporánea, específicamente sobre la pregunta: ¿La sociedad se sitúa frente un fenómeno que corresponde a desdiferenciación o radicalización de la diferenciación funcional?

Si hay un punto de inflexión en el que los estudios de teoría de sistemas han intentado diferenciarse de la sociología tradicional, ha sido en la búsqueda de precisión conceptual a la hora de enfrentar la investigación empírica (Luhmann 2007; 1997), siempre con el objetivo de intentar posicionar nuevas explicaciones plausibles. Un desafío que continúa y que, aun cuando las contingencias evolutivas de la sociedad lo pongan a prueba, no debe abandonarse. RM

\section{Bibliografía}

Arellano, C. (2010). Las Isapres ante el Tribunal Constitucional: Urgen reglas claras. CIPER. Consultado el 29 de junio de 2012, desde http:// ciperchile.cl/2010/ 05/28/las- isapres- ante- el- tribunal- constitucional- urgenreglas- claras.

Arnold, M. (2008). Las organizaciones desde la teoría de los sistemas sociopoiéticos. Cinta de Moebio, 32, 90- 108.

Barría, P. (2012). Alza de precios en los planes de ISAPREs: Seis verdades ocultas. CIPER. Consultado el 29 de junio de 2012, desde http:// ciperchile.cl/2012/04/05/alza- deprecios- de- los- planes- de- isapres- seis- verdades- ocultas/.

Brandão, G. (2012). Acerca del concepto de sistema: Desde la observación de la totalidad hasta la totalidad de la observación. Revista Mad, 26, 44- 53.

Cadenas, H. (2012). La desigualdad de la sociedad. Diferenciación y desigualdad en la sociedad moderna. Persona y Sociedad, 26(2), 51- 77.

Caviedes, R. (2012). Mañana será otro día [Emisión de Radio]. Entrevista a Rafael Caviedes, Director Ejecutivo de la Asociación de Isapres. Santiago de Chile: Radio Concierto. Consultado el 29 de junio de 2012, desde http:// www.concierto.cl/ 3555/ rafael- caviedes- director- ejecutivo- de- la- asociacionde-isapres/

CENDA. (2011). Financiamiento de la Educación Superior CENDA-CONFECH 2011. Consultado el 02 de Junio de 2012, desde http:// www.cendachile.cl/Home/ publicaciones/ temas/ reforma- educacional1 / financiamiento- de- la- educacion- superior- cenda- confech- 2011.

Eisenstadt, S. N. (1968). Modernización. Movimientos de protesta y cambio social. Buenos Aires: Amorrortu editores.

Fernández, M. (2011). Mejores alumnos de liceos públicos logran 139 puntos menos en la PSU que sus pares de colegios privados. Diario El Mercurio. Consultado el 29 de junio de 2012, desde http:/ / diario.elmercurio.com/ detalle/ index.asp?id=\{df40c08c-9d654fca- 923e-f16556d6192f\}.

Johansen, O. (2007). Teoría General de los Sistemas Sociales. Santiago de Chile: Universidad de Ciencias de la Información. 
Knudsen, M. (2007). Structural Couplings Between Organisations and Function Systems Looking at Standars in Health Care. Cybernetics \& Human Knowing, 14(2-3), 111131.

Kuhn, T. (2010). La estructura de las revoluciones científicas ( $3^{\mathrm{a}}$ ed.). México D. F.: FCE.

Leibniz, G. (1980). Monadología. Buenos Aires: Aguilar.

Ley $\mathrm{N}^{\circ} 19.496$ Establecer normas sobre protección de los derechos de los consumidores (1997, Marzo 7). Diario Oficial de la República de Chile. Consultado el 29 de junio de 2012, desde http:// www.sernac.cl/leyes/ compendio/ docs_compendio/Ley19496.pdf

Luhmann, N. (1994). Unidad y diferenciación en la sociedad moderna. Acta Sociológica, $12,55-61$.

Luhmann, N. (1997). La cultura como concepto histórico. Historia y Grafía, 8, 11- 33.

Luhmann, N. (1998a). La diferenciación de la sociedad. En N. Luhmann, Complejidad y Modernidad: De la unidad a la diferencia (pp. 71-99). Madrid: Trotta.

Luhmann, N. (1998b). Inclusión y Exclusión. En N. Luhmann, Complejidad y Modernidad: De la unidad a la diferencia (pp. 167-197). Madrid: Trotta.

Luhmann, N. (1998c). La forma "persona". En N. Luhmann, Complejidad y Modernidad: De la unidad a la diferencia (pp. 231-245). Madrid: Trotta.

Luhmann, N. (2000) Organisation und Entscheidung. Opladen: Westdeutscher Verlag.

Luhmann, N. (2005). Poder. México D.F.: Anthropos- PUC.

Luhmann, N. (2007). La Sociedad de la Sociedad. México: Herder.

Luhmann, N., \& Schorr, K. (1990). Presupuestos estructurales de una pedagogía reformista: Análisis sociológico de la pedagogía moderna Revista de Educación, 291, 55- 79.

Mascareño, A. (2005). La imposibilidad de la igualdad por la vía educativa. Asuntos Públicos, Centro de Estudios del Desarrollo. Consultado el 29 de junio de 2012, desde http:// www.ced.cl/ap/ wp- content/ uploads/2005/12/513.pdf

Mascareño, A. (2011) Sociología de la intervención: orientación sistémica contextual. Revista Mad, 25, 1- 33.

Nassehi, A. (2011). La teoría de la diferenciación funcional en el horizonte de sus críticas Revista Mad, 24, 1- 29.

Riveros, L. (2004). Columna: Inflación de notas. Diario Estrategia. Consultado el 29 de junio de 2012, desde http:// www.uchile.cl/portal/presentacion/ historia/ luis- riveroscornejo/ columnas/ 5416/inflacion- de- notas

Robles, F. (2002). Opciones de reinclusión para domiciliaridades dañadas. Espacio Abierto, $11(1), 9-24$.

Robles, F. (2006a). Hablo contigo si tú hablas conmigo: Metódica y análisis de los sistemas de interacción. Concepción: Escaparate.

Robles, F. (2006b). Autopoiesis, inclusión y tiempo: La indolencia ante la exclusión social. Revista de la Academia, 11, 91- 106.

Robles, F. (2010) Los ecos de la vergüenza: Pasado y presente de la exclusión social en Chile. Consultado el 28 de junio de 2012, desde http:/ / es.scribd.com/ doc/44343702/ Los- Ecos- de- La- Verguenza

Rodríguez, D. (2004). Organizaciones para la modernización. México D.F.: Universidad Iberoamericana.

Rodríguez, D. (2012). Un concepto sistémico de cultura organizacional. En H. Cadenas, A. Mascareño \& A. Urquiza (Eds.), Niklas Luhmann y el legado universalista de su teoría. Aportes para el análisis de la complejidad social contemporánea (pp. 393-404). Santiago de Chile: RIL editores.

Rodríguez, D. \& Arnold, M. (1991). Sociedad y teoría de sistemas. Santiago de Chile: Editorial Universitaria.

Spencer- Brown, G. (1972). Laws of Form (2a ed.). London: The Julian Press.

Torres Nafarrate, J. (2004). Luhmann: La política como sistema. México D.F.: FCE.

Ziemann, B. (2007). The Theory of Functional Differentation and the History Modern Society. Reflections on the Reception of Systems Theory in Recent Historiography. Soziale Systeme, $13(3), 220-229$. 


\section{Sobre los autores}

Julio Labraña es sociólogo de la Universidad de Concepción, Chile. Actualmente se desempeña como investigador en la Universidad Federico Santa María-Sede Concepción. Sus áreas de especialización son: Teoría sociológica, teoría de sistemas sociales, sociología de la educación, metodologías de la investigación social, políticas de intervención, desigualdad social.

jlabrana@ug.uchile.cl

Felipe Pérez- Solari es historiador de la Universidad de Concepción, Chile. Actualmente se desempeña como investigador en el Departamento de Historia de la Universidad de Concepción, Chile y como becario Coicyt, Chile. Sus áreas de especialización son: Teoría de Sistemas Sociales, sociología política, sociología histórica, teoría de la historia, historia moderna y contemporánea.

fperezsolari@ug.uchile.cl

Felipe Rivera es sociólogo de la Universidad de Concepción, Chile. Actualmente se desempeña como investigador en el Departamento de Educación, Fundación Integra, Chile. Sus áreas de especialización son: Teoría de sistemas sociales, fenomenología, etnometodología, análisis conversacional, estudios culturales, pedagogía crítica.

felirivera@ug.uchile.cl

Evelyn Campos es Periodista de la Universidad de Chile, Chile. Actualmente se desempeña como colaboradora en temas editoriales y comunicacionales y como investigadora en proyectos Fondecyt. Sus áreas de especialización son: Aspectos sociales de Inernet, videomúsica, semiótica de la percepción.

evelie.campos@gmail.com

Contacto

Universidad Técnica Federico Santa María- Sede Concepción

Alemparte 943 - Hualpén

Chile

Recibido: Junio 2012

Aceptado: Agosto 2012 